Questions vives

\section{Questions Vives}

Recherches en éducation

Vol. $7 n^{\circ} 14 \mid 2010$

TIC et développement des compétences : quelles réciprocités?

\title{
Poucet, B., La liberté sous contrat. Une histoire de l'enseignement privé
}

Paris : Fabert, 2009

\section{Rozenn Rouillard}

\section{(2) OpenEdition \\ Journals}

\author{
Édition électronique \\ URL : http://journals.openedition.org/questionsvives/701 \\ DOI : 10.4000/questionsvives.701 \\ ISBN : 978-2-8218-1088-4 \\ ISSN : $1775-433 X$ \\ Éditeur \\ Université Aix-Marseille (AMU) \\ Édition imprimée \\ Date de publication : 15 décembre 2010 \\ Pagination : 179-182 \\ ISBN : 978-2-912643-38-4 \\ ISSN : 1635-4079
}

Référence électronique

Rozenn Rouillard, « Poucet, B., La liberté sous contrat. Une histoire de l'enseignement privé », Questions Vives [En ligne], Vol. $7 n^{\circ} 14$ | 2010, mis en ligne le 15 juin 2011, consulté le 22 septembre 2020. URL http://journals.openedition.org/questionsvives/701; DOI : https://doi.org/10.4000/questionsvives. 701

Ce document a été généré automatiquement le 22 septembre 2020.

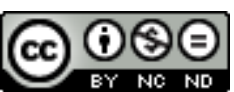

Questions Vives est mis à disposition selon les termes de la licence Creative Commons Attribution Pas d'Utilisation Commerciale - Pas de Modification 4.0 International. 


\section{Poucet, B., La liberté sous contrat. Une histoire de l'enseignement privé}

Paris : Fabert, 2009

Rozenn Rouillard

\section{RÉFÉRENCE}

Poucet, B. (2009). La liberté sous contrat. Une histoire de l'enseignement privé. Paris : Fabert.

\section{NOTE DE L'ÉDITEUR}

L'auteur, Bruno Poucet, est Professeur des universités en Sciences de l'éducation à l'université de Picardie Jules-Verne, membre du laboratoire Habiter-PIPS. Ses travaux portent particulièrement sur l'histoire de l'enseignement philosophique et de l'enseignement privé. Il est également rédacteur en chef de la revue Carrefours de l'éducation.

1 En France, l'histoire du système scolaire ne peut être appréhendée dans sa globalité sans porter un regard attentif et aiguisé sur celle de l'enseignement privé, comme notamment celui de Bruno Poucet depuis quelques années. Son dernier ouvrage s'attache à retracer les traits historiques du secteur d'enseignement associé à l'État, en l'occurrence catholique, avec une question centrale qui le guide : comment expliquer le présent par le passé ? Le résultat émane du travail imbriqué de l'historien et du chercheur en sciences de l'éducation. Objet en France de rares études aux orientations disciplinaires et théoriques diverses, l'enseignement privé se visite ici par un important dépouillement d'archives publiques et privées, de bulletins des organisations syndicales, d'ouvrages et d'articles. De la fin de la seconde guerre mondiale à aujourd'hui, l'auteur éclaire alors les processus de création de lois qui aboutissent ou qui échouent, en mettant souvent en évidence "l'officieux» afin d'expliquer «l'officiel » d'une histoire qui lie l'enseignement catholique au politique, à la société, 
l'État, l'Église et l'enseignement public. Il enrichit la réflexion autour de la construction d'une culture, celle de l'enseignement catholique. Les transformations institutionnelles du secteur privé d'enseignement, l'évolution de ses fonctions et de son usage ont un rapport dialectique avec le secteur public, ce qui conduit l'auteur à explorer les conditions de « rapprochement des deux cultures » (p. 105-106), dont la loi Debré figure en symbole.

2 Bruno Poucet nous invite dans un avant-propos à un balayage des premières années du $\mathrm{XX}^{\text {ème }}$ siècle marquées par la "guerre scolaire ", sous l'influence de groupes d'acteurs aux intérêts divergents: "on est d'un camp ou de l'autre» (p. 19). L'attention du chercheur se concentre dans les deux premiers chapitres sur les processus de rapprochement entre les deux secteurs d'enseignement après la fin de la Seconde Guerre mondiale. Ces processus se caractérisent à l'époque surtout par des échecs, notamment autour de projets gouvernementaux (par exemple, le projet Capitant en 1945), mais ces années de négociation entre des Hommes et de confrontations d'idées préparent assurément le vote de la Loi Debré en 1959. De plus, dans le projet d'ordonnance de René Capitant, se fait jour une distinction entre éducation et enseignement, « la question religieuse étant relative à l'éducation » (p. 36), préfigurant tout au long de l'histoire une caractérisation sectorielle différenciée, avec la primauté de l'éducation par rapport à l'instruction dans le privé, et inversement dans le public. Se profilent également, dans ces années 1950, deux éléments qui jouent, tout au long de l'histoire scolaire, des rôles importants : la nécessité du compromis quand chacun a besoin de l'autre (l'Etat a besoin de l'enseignement privé, et vice-versa, avec l'argent comme nerf de la guerre) et le poids des acteurs de toutes parts (syndicats, comités, fédérations, secrétariats, organismes, associations, mouvements), de personnalités politiques et de l'opinion publique. En 1951, la pression d'acteurs de l'enseignement catholique permet ainsi le vote des lois Marie-Barangé, qui représente un pas important, politiquement et socialement : «désormais, le principe même d'une aide à l'enseignement libre est acquis». L'enseignement catholique n'existe pourtant pas "comme un tout unifié » (p. 57) pendant la IV ème République. Il se structure, se centralise par la suite.

3 Les trois chapitres qui suivent, s'ouvrent à l'entrée dans la Vème République, avec une année-clé dans l'histoire de l'enseignement privé : 1959. Cependant, l'année démarre par l'échec d'une nouvelle ordonnance, miroir de deux écoles opposées, de deux cultures qui se construisent. « La loi d'aide à l'enseignement privé », nommée loi Debré, met alors en marche, selon l'auteur, " une véritable révolution culturelle» (p. 89) : collaboration, coopération avec l'enseignement public et association avec l'État. Cet acte politique se lit temporellement, en trois phases, car il s'est construit progressivement : temps de négociations entre déclarations et secrets, création de la commission Lapie et des conditions d'un compromis, avant-projet de loi BoullocheDebré et débats sur les termes. Le titre de cet avant-projet se voit modifié, symbolisant une victoire des deux camps et entraînant indirectement leur rapprochement. Le projet de loi est désormais "entre l'État et les établissements d'enseignement privés », alors qu'il portait précédemment sur les «rapports de l'état et des établissements privés d'enseignement ». L'État gagne en ne reconnaissant pas le secteur privé comme "une entité indépendante » (p. 83) : ce sont les établissements. Mais la victoire du privé se révèle dans le changement de place, et donc de signification, de l'enseignement : c'est «la reconnaissance du caractère spécifique de l'enseignement » (p.83) et une certaine 
mainmise sur l'enseignement par les écoles catholiques, qui ne sera néanmoins visible que dans le titre. L'article premier de la loi insiste sur la distinction entre éducation et instruction, et sur la célèbre expression du « caractère propre » de l'établissement sous contrat, dont les interprétations sont multiples. L'application de la loi entraîne ensuite une structuration centralisée de l'enseignement « libre » qui devient «catholique » en 1966. Les cultures en construction font débat dans les années 1960, et le rapprochement des deux fait parfois peur.

Les sixième et septième chapitres sonnent les années 1970, période pendant laquelle les deux parties se reconnaissent, mais le contexte s'avère complexe et fragile, entre « désaccord, un peu pour la forme» (p. 107), échanges secrets et accommodements. La spécificité de chaque culture autour de valeurs communes constitue toujours un enjeu essentiel pour chacun, avec du lobbying de chaque côté : « chacun joue sa partition sans plus de concertation » (p. 117). La loi de 1971 renforce la liberté d'enseignement et éloigne l'association de l'enseignement sous contrat au service public, à une période pendant laquelle le privé continue de s'organiser et se place au cœur du débat politique. Le pouvoir en place prend parti pour l'enseignement privé, avec l'argument privilégié de sa défense, à savoir la liberté d'enseignement : c'est la loi Guermeur en 1977 qui installe définitivement le secteur privé en France. Le huitième chapitre débute par l'arrivée de la gauche au pouvoir en 1981 qui scande la «mise en place d'un grand service public, unifié et laïque de l'Education nationale"; ce sera finalement un "retour à la logique de la loi Debré» (p. 141), "devenue désormais ligne jaune infranchissable» (p. 169). Pour les deux camps, il s'agit de passer du combat politique au combat social, dans une époque d'insuccès du projet de rapprochement des secteurs " privé » et « public » pour des questions identitaires notamment (autour du statut des enseignants du privé par exemple), de «bataille de l'opinion» (p. 154) et de grandes manifestations. Des «mesures simples et pratiques» apportent quelques évolutions, mettant principalement fin au mouvement engagé dans les années 1970.

5 Les deux derniers chapitres de l'ouvrage mettent l'accent sur l'association de l'enseignement privé à l'Etat, allant crescendo, avec les enseignants au cœur de cette dynamique (concours, formation), transversale à un système éducatif qui connaît une augmentation importante de ses effectifs dans le second degré. A l'aube des années 1990, le double secteur est intégré et " accepté par au moins un tiers, voire davantage, de la population » (p. 176). Cette décennie voit la reprise du dialogue entre le privé et le public : «s'esquisse un rapprochement entre des organisations qui avaient perdu toute occasion de dialoguer, de se comprendre et surtout de négocier» (p. 177). L'accord Lang-Cloupet, en 1992, confirme la logique de renforcement de l'association au service public. La même année, celle-ci se révèle également reconnue par l'Église, lors de la promulgation d'un nouveau «statut » de l'enseignement catholique par les évêques de France, marquant l'évolution et « le paradoxe d'une institution à la fois d'Église (de par son histoire) et de la nation (de par les liens organiques noués avec l'État) » (p. 183-184). Les stratégies politiques reprennent lors des tentatives de modifications de la loi Falloux. Toutefois, la définition de la place respective des deux écoles et de leurs liens, en toile de fond de cette question des investissements, s'apparente toujours à une prise de risque : entretenir l'association, la complémentarité et rejeter la concurrence. Les années 1990 voient le public aller vers le privé, et vice-versa: les établissements privés et publics «se rapprochent et se banalisent» (p. 197). Cet état se renforce ces dernières années. Avec la loi Censi de 2005, les enseignants du privé mettent leurs pas statutaires dans ceux de la sphère publique. Cet emboîtement avec l'Etat amène alors 
une insistance de la part de l'épiscopat sur l'adoption d'un amendement précisant que "l'enseignement leur est confié, dans le cadre de l'organisation arrêtée par le chef d'établissement, dans le respect du caractère propre de l'établissement ». La place de l'enseignement privé a indéniablement changé : il se définit aujourd'hui davantage comme "un enseignement associé ouvert à tous les horizons spirituels ", qu'à "un enseignement confessionnel associé »(p. 201). Il s'agit de se concentrer sur la complémentarité entre les deux écoles, d'ailleurs au centre d'une loi de 2009, qui « lie le financement à un besoin scolaire reconnu» (p. 212). Les représentants de l'enseignement catholique veillent néanmoins à ce que le «caractère propre » ne soit pas vide de sens.

Dans la conclusion de l'ouvrage, l'auteur met en exergue le poids de la loi Debré dans cette histoire du processus de rapprochement de deux cultures, et de leur construction respectivement liée. Il fait remarquer son atypisme, son symbole, ses évolutions, représentations et effets. Les définitions identitaires sont ébranlées en ce début de nouvelle décennie. La constitution d'un ensemble scolaire sectorisé interroge actuellement, car le clivage privé-public se révèle de moins en moins significatif.

Bruno Poucet donne une réponse historique à ce rapprochement progressif des établissements privés et publics, avec une brillante minutie dans les noms et la chronologie des faits ou des moments qui marquent « une réalité faite de strates » (p. 209). Cette force devient parfois une faiblesse, car le lecteur peut se perdre dans les détails. Quant à l'histoire de la guerre scolaire, il aurait été apprécié d'avoir une rétrospective conséquente du XIX ${ }^{\text {ème }}$ siècle, afin notamment de prendre la mesure de l'opposition privé-public qui s'est construite pendant ce siècle. De plus, l'auteur aurait peut-être pu adopter un regard local, plus présent et plus marqué, à certains moments, tant on connaît le caractère régionalisé de l'enseignement catholique sur le territoire national. Par ailleurs, à travers quelques mots imprégnés d'un certain habitus, on devine l'auteur-acteur d'une partie de l'histoire qu'il semble avoir vécue en tant qu'enseignant du privé. L'engagement se ressent, n'allant pas à l'encontre d'un travail objectivé, nourrissant même une vue de l'intérieur de la construction d'une «liberté sous contrat ». Ce titre de l'ouvrage est éloquent, spécialement par le lien inextricable qu'il symbolise : le privé ou l'enseignement libre et l'État ou le politique. Bruno Poucet esquisse l'histoire de ce lien par un tableau d'ensemble, tout autant rigoureux et inédit que passionnant.

\section{AUTEURS}

\section{ROZENN ROUILLARD}

Sciences de l'éducation - CREAD - Université de Rennes 2 